\title{
Final Report for DE-FC02-01ER25473: \\ "An Algorithmic and Software Framework for Applied Partial Differential Equations (APDEC): A DOE SciDAC Integrated Software Infrastructure Center (ISIC)"
}

\author{
Professor Elbridge Gerry Puckett \\ Department of Mathematics \\ University of California, Davis \\ Davis, CA, 95616
}

May 13, 2008

\section{Introduction}

All of the work conducted under the auspices of DE-FC02-01ER25473 was characterized by exceptionally close collaboration with researchers at the Lawrence Berkeley National Laboratory (LBNL). This included having one of my graduate students - Sarah Williams - spend the summer working with Dr. Ann Almgren a staff scientist in the Center for Computational Sciences and Engineering (CCSE) which is a part of the National Energy Research Supercomputer Center (NERSC) at LBNL. As a result of this visit Sarah decided to work on a problem suggested by Dr. John Bell the head of CCSE for her PhD thesis, which she finished in June 2007. Writing a $\mathrm{PhD}$ thesis while working at one of the University of California (UC) managed DOE laboratories is a long established tradition at the University of California and I have always encouraged my students to consider doing this. For example, in 2000 one of my graduate students - Matthew Williams - finished his PhD thesis while working with Dr. Douglas Kothe at the Los Alamos National Laboratory (LANL). Matt is now a staff scientist in the Diagnostic Applications Group in the Applied Physics Division at LANL.

Another one of my graduate students - Christopher Algieri - who was partially supported with funds from DE-FC02-01ER25473 wrote am MS 
Thesis that analyzed and extended work published by Dr. Phil Colella and his colleagues in 1998. Dr. Colella is the head of the Applied Numerical Algorithms Group (ANAG) in the National Energy Research Supercomputer Center at LBNL and is the lead PI for the APDEC ISIC which was comprised of several National Laboratory research groups and at least five University PI's at five different universities. Chris Algieri is now employed as a staff member in Dr. Bill Collins' research group at LBNL developing computational models for climate change research. Bill Collins was recently hired at LBNL to start and be the Head of the Climate Science Department in the Earth Sciences Division at LBNL. Prior to this he had been a Deputy Section Head at the National Center for Atmospheric Research in Colorado. My understanding is that Chris Algieri is the first person that Bill hired after coming to LBNL. The plan is that Chris Algieri will finish his $\mathrm{PhD}$ thesis while employed as a staff scientist in Bill's group.

Both Sarah and Chris were supported in part with funds from DE-FC0201ER25473. In Sarah's case she received support both while at U.C.!Davis (UCD) taking classes and writing an MS thesis and during some of the time she was living in Berkeley, working at LBNL and finishing her $\mathrm{PhD}$ thesis. In Chris' case he was at U.C. Davis during the entire time he received support from DE-FC02-01ER25473. More specific details of their work are included in the report below.

Finally my own research conducted under the auspices of DE-FC0201ER25473 either involved direct collaboration with researchers at LBNL - Phil Colella and Peter Schwartz who is a member of Phil's Applied Numerical Algorithms Group - or was on problems that are closely related to research that has been and continues to be conducted by researchers at LBNL. Specific details of this work can be found below.

Finally, I would like to note that the work conducted by my students and me under the auspices of this contract is closely related to work that I have performed with funding from my DOE MICS contract DE-FC02-03ER25579 "Development of High-Order Accurate Interface Tracking Algorithms and Improved Constitutive Models for Problems in Continuum Mechanics with Applications to Jetting" and with my CoPI on that grant Professor Greg Miller of the Department of Applied Science at UCD. In theory I tried to use funds from the SciDAC grant DE-FC02-01ER25473 to support work that directly involved implementing algorithms developed by my research group at U.C. Davis in software that was developed and is maintained by my SciDAC CoPI's at LBNL. For example, see the work reported in Sections 2 and 4 below. However, since there is considerable lead time spent developing such algorithms before they are ready to be implemented in lab codes, (e.g., 
as in section 4) or research plans and goals change (e.g., as in sections 2 and 3, I have supported each member of my research group partially with funds from the SciDAC APDEC ISIC DE-FC02-01ER25473 and partially with funds from my DOE MICS grant DE-FC02-03ER25579. This has resulted in a significant overlap of projects areas that are funded by both grants.

\section{Development of a Fast Multipole Method-based Poisson Solver with Applications to Approxi- mating Solutions of the Incompressible Euler and Navier-Stokes Equations}

Under my guidance Sarah Williams implemented an algorithm for approximating solutions of the Poisson equation

$$
\Delta \phi=f
$$

that is based on the Fast Multipole Method (FMM) [14]. Williams' work is based on previous work by Greengard and his collaborators [10, 13].

The Poisson equation (1) is ubiquitous in science and engineering and the numerical solution of the Poisson equation is often (almost always!) the most expensive and least robust part of numerical algorithms used in science and engineering applications. In particular, in a projection method for approximating solutions of the incompressible Euler or incompressible Navier-Stokes equations one must solve a version of (1) one or more times per timestep. The original projection method is due to Chorin [8] and much of the development of modern, high-order versions of these methods is due to Phil Colella and John Bell and their coworkers at LBNL (e.g., [2, 3, 4, 6]).

Typically these researchers use some form of iterative Poisson solver (e.g., MultiGrid (MG) or PreConditioned Conjugate Gradient (PCCG)) in their projection methods. However, for some problems iterativemethods can take an unreasonably large number of iterations to converge, or even fail to converge. The goal of Sarah's work was to develop a direct rather than iterative solver that is guaranteed to produce an accurate approximation to the solution of (1) in a fixed amount of time that can can be determined $a$ priori, even when iterative methods fail.

In Sarah's Masters thesis [27] she describes her implementation of the algorithm and compared the efficiency of this algorithm to the efficiency of MG on a class of test problems she devised. 
Sarah spent the summer of 2005 working with Dr. Ann Almgren in the Center for Computational Sciences and Engineering (CCSE) at LBNL implementing her method in the FORTRAN 90 software infrastructure that CCSE researchers have developed and examining the performance of this method on problems for which the MultiGrid solver that CCSE researchers typically use in their projection method either fails, or uses an unusually large number of iterations. This work is reported on in the following LBNL Technical Report [29].

\section{Numerical Methods for the Stochastic Landau- Lifshitz Navier-Stokes Equations}

By the end of her stay at LBNL in the summer of 2006 working with Dr. Almgren and interacting with Dr. Almgren's coworkers at CCSE Sarah decided that she wanted to change the direction of her research for her $\mathrm{PhD}$ thesis. She liked the research environment at LBNL and elected to stay there and work on a topic suggested by Dr. John Bell, namely the development of numerical methods for the stochastic Landau-Lifshitz Navier-Stokes Equations. Under the guidance of Dr. Bell and his collaborator Professor Alejandro L. Garcia from San Jose State University Sarah wrote a PhD thesis entitled "Algorithm refinement for the stochastic Navier-Stokes equations" that was filed in June 2007 [28]. During the first year of her work on her PhD thesis (i.e.; Academic Year 2005-2006) I supported Sarah with funds from both DE-FC02-03ER25579 and DE-FC02-01ER25473. During her second and final year at LBNL, she was supported with a Dissertation Year Fellowship from the University of California, Davis and a $\$ 10,000$ scholarship known as a PEO Scholarship. Sarah is currently a Postdoctoral Research Associate in Applied Mathematics at UNC Chapel Hill. The following publications have resulted from her work ([7, 26]).

\section{Analysis of algorithms for tracking material in- terfaces that are second-order accurate in time}

Under my guidance Chris Algieri completed an MS thesis in December 2005 in which he examined algorithms for tracking the motion of a material interface that are second-order accurate in time [1]. This is currently an extremely active area of research In particular, our ANAG colleagues at LBNL have recently published several papers in this area (e.g., [9, 19, 24]). 
In his work Algieri studied the conditions under which an algorithm that is based on a second-order accurate stencil of the spatial derivatives fails to be second-order accurate in time when used with standard second-order accurate time stepping algorithms such as Crank-Nicholson. This problem was first noticed by Johansen and Colella in [18]. Their solution involved using a more stable time stepping algorithm due to Twizell, Gumbell and Arigu [25]. In his MS thesis [1] Chris studied algorithms for numerically analyzing the stability of operators that are based on second-order spacial stencils and determined criteria for deciding when a method will be secondorder accurate in time. Chris' work focused on the numerical solution of the Stefan problem; i.e., the heat equation with an interface at temperature $T=0$ at which the material in question solidifies.

\section{Development and Analysis of the High-Order Accurate Volume-of-Fluid Interface Reconstruc- tion Methods}

During the funding period covered by DE-FC02-01ER25473 I revised and published a paper in which my former PhD student James Pilliod and I developed a new class of second-order accurate volume-of-fluid (VOF) interface reconstruction algorithms [22]. ${ }^{1}$ We called the simplest and most efficient of these second-order accurate interface reconstruction algorithms the "Efficient Least Squares VOF Interface Reconstruction Algorithm" (ELVIRA). Some researchers in this area now refer to any VOF interface reconstruction algorithm that uses the same basic idea as belonging to the ELVIRA class or family of algorithms.

In any numerical method for modeling the motion of the interface between two materials one generally needs two distinct algorithms. First one must have a numerical representation of the interface itself at some fixed moment in time. A method for doing this is called an interface reconstruction algorithm. Then, if the interface is moving in time, one must also have an algorithm for tracking or advecting the interface in time. In [22] Pilliod and I also introduced a new second-order accurate volume-of-fluid advection algorithm that was based on ideas developed by John Bell and his coauthors in [5].

\footnotetext{
${ }^{1}$ All of Pilliod's work predated the period covered by DE-FC02-01ER25473. After finishing his $\mathrm{PhD}$ thesis Pilliod spent several years as a PostDoc in Phil Colella's group at LBNL and then took a job in the "dot.com" industry. However I was partially supported by DE-FC02-01ER25473 while I was revising [22] for publication.
} 
Volume-of-fluid interface tracking algorithms are important for a variety of reasons. From DOE's - and the nation's - point of view perhaps the most important reason is that most - if not all - of the "production" codes that are used by scientists at the Los Alamos National Laboratory and the Lawrence Livermore National Laboratory to model the movement of a large number of distinct materials are based on some version of a volume-of-fluid algorithm. For example, there are codes that can model dozens of different materials in which each material is tracked by keeping a record of the volume fraction in each cell that is occupied by that material. There are competing interface tracking methods - most notably the level set method - but to the best of my knowledge there are currently no production codes that can model the movement of a large number of distinct materials that are based on some version of the level set method, or any method other than some version of a volume-of-fluid method.

In all of the work with Pilliod in [22] we demonstrated that our algorithms were second-order accurate by making multiple computations of a given problem on successively refined grids, each grid a factor of two finer than the grid which we had used in the previous computation. This allowed us to compute the convergence rate and hence the accuracy of the algorithm for that particular problem. However, except in certain limited instances, we did not offer a proof that any of our algorithms were second-order accurate.

Recently, in collaboration with Professor Greg Miller of the Department of Applied Science at U.C. Davis, I have proven that the ELVIRA family of interface reconstruction algorithms are second-order accurate [20]. This work was partially supported by DE-FC02-01ER25473.

There are several other interesting and important results in [20]. First, we prove that a VOF interface reconstruction algorithm is second-order accurate provided that

1. The volume of each material in the cell is exact.

2. The slope of the line that one uses to represent the interface in the cell is first-order accurate.

A corollary of this theorem is that if the slope of the line that represents the interface in the cell is less than first-order accurate (i.e., zeroth-order accurate), then the linear representation of the interface in the cell is firstorder accurate. This implies that SLIC [21] - which is probably the best known and most widely used VOF method - is first-order accurate. This fact has been demonstrated numerous times in convergence rate studies (e.g., in [22]), but to the best of my knowledge has never been proven. One 
reason it is important to understand the accuracy of SLIC and volume-offluid interface reconstruction algorithms similar to SLIC, is that they are widely used to model problems in which one of the materials breaks up into very small pieces in which the volume of the individual pieces is on the order of the volume of a computational cell; i.e., the volume of the drops or pieces of one or more of the materials being modeled is on the order of $(\Delta x)^{3}$ where $\Delta x$ represents the local grid spacing. For example, in [15] my coauthors and I used this algorithm (SLIC) and in [16] and [23] we used a hybrid ELVIRA-SLIC algorithm in which we used a second-order accurate ELVIRA algorithm except when the volume of one of the materials being tracked approached $(\Delta x)^{3}$, in which case we reverted to SLIC.

A second important corollary of the work in [20] is that our proof that certain VOF interface reconstruction methods are second-order accurate contains a constant that relates the error to the local curvature $\kappa$ of the interface in such a way that if the grid spacing $\Delta x$ is too large as compared to the radius of curvature $R \equiv 1 / \kappa$, then the observed convergence rate will be first-order rather than second-order. In other words, we have found a criterion for predicting when the computation of the interface will be underresolved, namely when

$$
\Delta x>>\frac{1}{6 \kappa} .
$$

The phenomenon of an underresolved volume-of-fluid computation of an interface is observed and discussed in e.g., [22]. One important way in which one can use (2) is as a refinement criterion when one is using a block structured adaptive mesh refinement method to model the motion of an interface. In this case one can compute an approximation to the curvature of the interface in each cell and then apply equation (2) in each cell to determine if the computation is underresolved in that cell. Cells in which the interface is found to be underresolved are tagged for refinement.

Finally I'd like to note that as computational models are increasingly being used for major public policy decisions (e.g., whether to build a peripheral canal in California, how high and strong levees must be in order to protect housing in California's Central Valley under worst case scenario conditions, etc.), having objective criteria for when a computation is well-resolved as opposed to underresolved is an increasingly important issue.

Professor Greg Miller and I have submitted [20] for publication and have received the reports of three referees. We are currently revising the paper and expect it to be published soon. 


\section{Analysis of Two Competing Methods for Mod- eling Problems in which the Motion of the In- terface Depends on the Normal Derivative}

In some problems involving the motion of the interface between two materials the speed of the interface depends on conditions at the interface itself. The classic example is the Stefan problem, which is a model for the solidification or melting of some material such as water and ice. In the Stefan problem the speed of the interface is proportional to the jump in the derivative of the temperature normal to the interface

$$
s=C \frac{\partial T}{\partial n}
$$

Here $s$ represents the speed of the front, $T$ the temperature of the water (say), $n$ is the unit normal to the front and $C$ is a constant that depends on the thermodynamic properties of the material being modeled.

The Stefan problem is usually posed as follows. Given initial conditions at some time $t=0$ the problem is to:

1. Determine the location of the interface between water and ice at each time $t>0$.

2. Determine the temperature $T$ at each point $x$ and time $t>0$.

In a numerical method to solve the Stefan problem in which the front is being tracked with some interface tracking method one needs an accurate approximation to the speed of the front in order accurately model the motion of the front. Since by equation (3) the speed of the front depends on the normal derivative of the temperature at the front, it is apparent that in order to accurately model the location of the front in time, one must first have an accurate numerical approximation to the normal derivative of the temperature at the front. In his $\mathrm{PhD}$ thesis [17] Hans Johansen proposed a method for doing this that results in a second-order accurate approximation to the normal derivative at the front. I will refer to this method as Colella and Johansen's (CJ) method. Their work is published in [18] and [19].

The price that one pays to achieve second-order accuracy of the normal derivative in CJ's method is that their finite difference stencil leads to a non-symmetric matrix $A$, where $A$ is the numerical approximation to the Laplace operator $\Delta$ that appears in the heat equation

$$
\frac{\partial T}{\partial t}=C_{1} \Delta T
$$


that must be solved in order to update the temperature $T$ in time. In order to obtain a second-order accurate approximation to the temperature $T$ as it evolves in time (e.g., with Crank-Nicholson) one must solve systems of the form

$$
A x=b .
$$

Since the numerical solution of (5) is typically easier to implement and faster when $A$ is symmetric, some researchers have sought alternatives to CJ's method.

In particular, in [12] the authors propose an alternative approximation to the normal derivative that results in a symmetric matrix $A$. I will refer to their method as Gibou and Fedkiw's (GF's) method. In [12] the authors demonstrate with numerical examples that their method yields second-order accurate solutions to the static (time independent) problem (1) with boundary conditions given on the normal derivative of the solution at the boundary; i.e., the Poisson problem with Neumann boundary conditions.

They then present examples of numerical solutions to the Stefan problem using their symmetric discretization at the interface which demonstrate that their algorithm is only first-order accurate when one uses their approximation the normal derivative at the interface

$$
s=C \frac{\partial T}{\partial n}
$$

to compute the speed of the interface at each time step. However, the authors offer no explanation for this decrease in accuracy when their method is used to compute solutions of the Stefan problem. In a later paper [11] Gibou and Fedkiw present a fourth-order accurate version of their algorithm and demonstrate that it yields third order accuracy when they use it to compute several examples of the Stefan problem. However, again they offer no explanation for the decrease in accuracy when one uses their algorithm to compute the speed of the interface.

I have recently completed an analysis of Gibou and Fedkiw's original algorithm from [12] in which I prove that their discretization of the normal derivative of the solution at the interface is first-order accurate not secondorder accurate. My analysis is based on the analysis Johansen used in his $\mathrm{PhD}$ thesis [17] to prove that the CJ discretization yields a second-order accurate approximation to the normal derivative of the solution to the Stefan problem and hence to the speed of the moving interface in the Stefan problem.

Although the problem that both Johansen and I consider is the simplest case, namely the 1D version of the Stefan problem (where the interface is 
reduced to a point) it still has implications for discretizing the Stefan problem and two and three dimensions. For example, if one had a discretization of the normal derivative that was second-order accurate in two or three dimensions, then it must also be second-order accurate when one uses it to model the 1D Stefan problem embedded in two or three dimensions. Hence one is forced to conclude that the GF algorithm will be first-order accurate when one uses it to model the Stefan problem in any dimension. Furthermore, given that there are a limited number of symmetric discretizations of the normal derivative at the interface, one may also conclude that in order to construct a second-order accurate finite difference solution of the Stefan problem, one must necessarily solve a non-symmetric system of equations of the form (5).

This has implications for a variety of other important problems in fluid mechanics and computational physics that involve the movement of an interface between two materials. For example, the computation of the solution to the Euler or Navier-Stokes equations with two fluids with surface tension at the moving interface between these materials involves the computation of the normal to the interface and the jump in the value of the pressure at the interface. Arguments similar to the one I have just described are likely to show that one must solve a non-symmetric system of the form (5) in order to obtain second-order accurate computations of this problem. Just as with the Stefan problem, this problem is ubiquitous in science and industry.

Since at the present time, "state-of-the-art" algorithms for modeling many important problems in science and industry are at best second-order accurate, the analysis that I have just described can serve as a simple guide for researchers to use in determining when their computational model may be second-order accurate and when it has no possibility of being better than first order accurate.

The work described above was done during the 2005-2006 Academic Year with partial support from DE-FC02-01ER25473. I am currently in the process of writing it up for publication.

\section{Development of a second-order accurate method for modeling solutions of the Stokes equation with material interfaces}

In collaboration with Peter Schwartz and Phil Colella of the Applied Numerical Algorithms Group (ANAG) at the Lawrence Berkeley National Laboratory (LBNL) I have been developing a second-order accurate method for 
modeling solutions of the Stokes equations for problems that contain one or more moving inclusions across which the fluid properties (e.g., density and viscosity) may change. Problems with these features occur in geophysical models of the Earth's structure between the Core-Mantle Boundary and the surface of the earth and in a variety of biological models, such as the movement of very small creatures in a fluid (e.g., 'swimming' microbes or bacteria). The goal of this work is to design and develop an algorithm that will be implemented in the EBChombo software infrastructure that is being developed by ANAG researchers at LBNL.

My intention is for the resulting software to be a computational tool for of modeling these problems that is capable of handling arbitrary boundary geometries with adaptive mesh refinement that would be freely available to the general scientific community. Software that is based on block structured adaptive mesh refinement and state-of-art algorithms for tracking the interface between two materials such as described in some of the previous sections of this report does not, for example, exist in the geophysics community. Some of the geophysical applications of this work has been in collaboration with Professor Louise Kellogg of the U.C. Davis Geology Department and Professor Michael Manga of the U.C. Berkeley Geophysics Department.

My current goal is to implement the algorithm in the EBChombo software library during the summer of 2008 and have a paper describing the algorithm ready for publication by November 2008 .

\section{References}

[1] C. A. Algieri. A comparison of two methods to solve the 1-D heat equation on an irregular domain in the context of the Stefan problem. Ms thesis, Department of Mathematics, University of California, Davis, December 2005.

[2] A. S. Almgren, J. B. Bell, P. Colella, and T. Marthaler. A Cartesian Grid Projection Method for the Incompressible Euler Equations in Complex Geometries. SIAM J. Sci. Comput., 18(5):1289-1309, September 1997.

[3] A. S. Almgren, J. B. Bell, P. Colella, L. H. Howell, and M. L. Welcome. A Conservative Adaptive Projection Method for the Variable Density Incompressible Navier-Stokes Equations. J. Comput. Phys., 142(1):146, 1999. 
[4] J. B. Bell, P. Colella, and H. M. Glaz. A Second-Order Projection Method for the Incompressible Navier-Stokes Equations. J. Comput. Phys., 85:257-283, 1989.

[5] J. B. Bell, C. N. Dawson, and G. R. Shubin. An Unsplit, Higher Order Godunov Method for Scalar Conservation Laws in Multiple Dimensions. J. Comput. Phys., 74:1-24, 1988.

[6] J. B. Bell and D. L. Marcus. A Second-Order Projection Method for Variable Density Flows. J. Comput. Phys., 101:334-348, 1992.

[7] J. B. Bell, A. L. Garcia, and S. A. Williams. Numerical methods for the stochastic Landau-Lifshitz Navier-Stokes equations. Physical Review E, 76(016708):016708-1-12, 2007.

[8] A. J. Chorin. Numerical Solution of the Navier-Stokes Equations. Math. Comput., 22(3):742-762, 1968.

[9] P. Colella, D. Graves, and J. A. Greenough. A second-order method for interface reconstruction in orthogonal coordinate systems. Technical Report LBNL-45244, Lawrence Berkeley National Laboratory, January 2002.

[10] F. Etheridge and L. Greengard. A new fast fast multipole accelerated Yukawa solver in two dimensions. SIAM J. Sci. Comput., 23(3):741$760,2001$.

[11] F. Gibou and R. P. Fedkiw. A fourth order accurate discretization for the Laplace and heat equations on arbitrary domains, with applications to the Stefan problem. J. Comput. Phys., 202:577601, 2005.

[12] F. Gibou, R. P. Fedkiw, L.-T. Cheng, and M. Kang. A Second-OrderAccurate Symmetric Discretization of the Poisson Equation on Irregular Domains. J. Comput. Phys., 176:205-227, 2002.

[13] L. Greengard and J. Y. Lee. A direct adaptive Poisson solver of arbitrary order accuracy. J. Comput. Phys., 125(2):415-424, May 1996.

[14] L. Greengard and V. Rockhlin. Fast Multipole Method. J. Comput. Phys., 162(1):1-32, July 2000.

[15] L. F. Henderson, P. Colella, and E. G. Puckett. On the Refraction of Shock Waves at a Slow-Fast Gas Interface. J. Fluid Mech., 224:1-27, 1991. 
[16] L. F. Henderson and E. G. Puckett. Anomalous Refraction of Shock Waves in Materials with General Equations of State Part I. The Shock Pair System. Proc. Royal Soc. London A, 2008. (accepted).

[17] H. S. Johansen. Cartesian grid embedded boundary finite difference methods for elliptic and parabolic partial differential equations on irregular domains. Phd thesis, Department of Mechanical Engineering, University of California, Berkeley, Fall 1997.

[18] H. S. Johansen and P. Colella. A Cartesian Grid Embedded Boundary Method for Poisson's Equation on Irregular Domains. J. Comp. Phys., 147:60-85, 1998.

[19] P. McCorquodale, P. Colella, and H. S. Johansen. A Cartesian grid embedded boundary method for the heat equation on irregular domains. J. Comp. Phys., 173:620-635, 2001.

[20] G. H. Miller and E. G. Puckett. On the second-order accuracy of the (E)LVIRA family of interface reconstruction algorithms. J. Comput. Phys. submitted.

[21] W. F. Noh and P. R. Woodward. SLIC (Simple Line Interface Calculation). In Lecture Notes in Physics, A. I. van der Vooren and P. J. Zandbergen, editors, volume 59, pages 330-340. Springer-Verlag, New York, 1976.

[22] J. E. Pilliod and E. G. Puckett. Second-order accurate volume-offluid algorithms for tracking material interfaces. J. Comput. Phys., 199(2):465-502, 2004.

[23] E. G. Puckett and L. F. Henderson. The Anomalous Refraction of Shock Waves in Materials with General Equations of State Part II. Anomalous Refraction Wave Systems. Proc. Royal Soc. London A, 2008. (accepted).

[24] P. O. Schwartz, M. Barad, P. Colella, and T. J. Ligocki. A Cartesian grid embedded boundary method for the heat equation and Poisson's equation in three dimensions. J. Comput. Phys., 2005. to appear.

[25] E. H. Twizell, A. B. Gumel, and M. A. Arigu. Second-order $L_{0}$-stable methods for the heat equation with time-dependent boundary conditions. Adv. Comp. Math., 6:333-352, 1996. 
[26] S. A. Williams, J. B. Bell, and A. L. Garcia. Algorithm refinement for fluctuating hydrodynamics. Multiscale Modeling and Simulation, 6(4):1256-1280, February 2008.

[27] S. A. Williams. A comparison of a fast multipole method-based Poisson solver to a multigrid method. M. S. Thesis, University of California, Davis, December 2004.

[28] S. A. Williams. Algorithm refinement for the stochastic Navier-Stokes equations. PhD thesis, University of California, Davis, June 2007.

[29] S. A. Williams, A. S. Almgren, and E. G. Puckett. On Using a Fast Multipole Method-based Poisson Solver in an Approximate Projection Method. Technical report, Lawrence Berkeley National Laboratory, March 2006. LBNL-59934. 